\title{
Perspective
}

PERSPECTIVE Actualité en histoire de l'art

$1 \mid 2020$

Japon

\section{Éloge du primitivisme : d'autres visages de la peinture japonaise prémoderne}

In Praise of Primitivism: Other Faces of Premodern Japanese Painting

Ein Loblied auf den Primitivismus: Andere Gesichter der vormodernen japanischen Malerei

Elogio del primitivismo: altri aspetti della pittura giapponese premoderna

Elogio del primitivismo: otros rostros de la pintura japonesa premoderna

\section{Christophe Marquet}

\section{(2) OpenEdition}

Journals

Édition électronique

URL : http://journals.openedition.org/perspective/18718

DOI : $10.4000 /$ perspective. 18718

ISSN : 2269-7721

Éditeur

Institut national d'histoire de l'art

Édition imprimée

Date de publication : 5 juin 2020

Pagination : 221-236

ISBN : 978-2-917902-89-9

ISSN : $1777-7852$

Référence électronique

Christophe Marquet, «Éloge du primitivisme : d'autres visages de la peinture japonaise prémoderne », Perspective [En ligne], 1 | 2020, mis en ligne le 30 décembre 2020, consulté le 25 janvier 2021. URL http://journals.openedition.org/perspective/18718; DOI : https://doi.org/10.4000/perspective.18718 


\title{
Éloge du primitivisme : d'autres visages de la peinture japonaise prémoderne
}

\author{
Christophe Marquet
}

\author{
À la mémoire de Pascal Griolet \\ (1946-2020) \\ Je n'éprouve pas qu'un simple intérêt pour ces peintures de peu de valeur, ces peintures \\ grossières ; je perçois une grande " saveur " dans le savoir-faire sans technique \\ de ces artisans qui paraissent malhabiles, et je salue profondément leur absence totale \\ de démonstration de virtuosité ${ }^{\text {. }}$
}

L'intensification des relations entre le Japon et l'Occident à partir de la Restauration de Meiji en 1868 a conduit, dans de nombreux domaines, à la formation de concepts et de modes de pensée empruntés à l'Europe moderne. Il en va ainsi dans celui de la création artistique - comme est récemment venue le rappeler l'exposition Meiji : splendeurs $d u$ Japon impérial au musée Guimet ${ }^{2}$-, avec la formation du concept de bijutsu ( " beauxarts »), la compilation d'une histoire officielle de l'art national et l'institutionnalisation des pratiques artistiques. Cette réorganisation conceptuelle conduisit notamment à établir une distinction nette et hiérarchique entre art noble - l'art pour l'art produit par le génie créateur - et art manufacturier issu de la technique, et à forger une vision orthodoxe de l'art pictural et de son histoire.

Ainsi, au cours du processus de modernisation et de normalisation de Meiji, les autorités écartèrent certains genres des salons officiels de peinture - comme la peinture satirique, l'imagerie populaire d'Ōtsu (Ōtsu-e) ou la peinture érotique - sous prétexte qu'ils ne possédaient pas les " qualités " prétendument requises par la définition occidentale des beaux-arts et qu'ils s'apparentaient à des peintures " grotesques " d'un Japon rustique qui devait être refoulé3. Ces œuvres, dont les Japonais étaient pourtant jusqu'alors friands, étaient devenues incompréhensibles, incongrues même, et n'avaient pas leur place dans la " sphère publique " du monde moderne.

Il faudra attendre les années 1920 pour qu'une lecture critique de cette période de Meiji s'engage et permette de redécouvrir et de valoriser des formes picturales prémodernes à caractère "primitif ", c'est-à-dire ne répondant pas aux canons esthétiques dominants, en rupture avec les codes lettrés ou savants, et issues en partie de traditions rurales. Autrement dit un ensemble de productions picturales à dimension parfois artisanale, qui avait été exclu du cadre de la définition occidentalo-centrée des beaux-arts, établie 
au Japon dans le dernier tiers du XIX siècle. Précisons que ce terme " primitif " renvoie, dans les textes japonais convoqués dans cet essai, à des réalités de nature variée : tantôt il désigne la "période primitive " (shoki) et souvent négligée d'un genre artistique (par exemple les "ukiyo-e primitives »), parfois il correspond à un jugement de valeur esthétique, porté par des termes comme genshi-teki ("primaire ") ou soboku ("brut ", " naturel »).

Le primitivisme est, on le sait, une « invention moderne » de l'Occident, pour reprendre le titre du récent ouvrage de Philippe Dagen ${ }^{4}$, et même une fiction, issue des premiers temps de l'ethnologie et née de la situation coloniale, avant de pénétrer la sphère des avant-gardes artistiques au début du XXe siècle et de faire, aujourd'hui, l'objet d'une nécessaire révision critique. Notre propos n'est pas de procéder ici à la déconstruction de ce concept, mais de montrer comme la découverte et l'appréciation du "primitivisme " dans l'art japonais au cours du XX ${ }^{e}$ siècle et du début du XXI ${ }^{\text {e }}$ siècle - en décalage avec le courant dominant de l'histoire de l'art académique -, ont été le dénominateur commun de plusieurs moments de rupture que nous proposons ici de retracer, depuis le mouvement pour les arts populaires dans les années 1920, jusqu'aux récentes expositions sur la peinture "naïve », en passant par la redécouverte des peintres excentriques de l'époque d'Edo dans les années 1970. Cette analyse permet d'apprécier d'autres regards qui ont été portés au Japon même sur l'art prémoderne, par des intellectuels, des historiens de l'art et des conservateurs, et de proposer une vision plurielle qui enrichit notre appréciation des productions picturales de cette période, en remettant en cause le cadre et le corpus académiques définis à la fin du XIX ${ }^{e}$ siècle. Nous évoquerons aussi les écueils de cette vision des choses.

\section{La reconnaissance de la " peinture populaire " : le combat des années 1920}

L'un des premiers acteurs de cette relecture fut le penseur Yanagi Muneyoshi (ou Sōetsu, 1889-1961), qui entama dans les années 1920 une réflexion sur la nature de la production artistique de son pays et suscita un large mouvement de reconnaissance de ce qu'il baptisa le Mingei ou les " arts populaires ". Il est intéressant de revisiter aujourd'hui cette entreprise majeure de relecture de l'histoire des arts du Japon - qui englobe une nouvelle conception de la peinture, sous le néologisme minga (" peinture populaire ") forgé en 1927 -, et d'examiner son héritage.

Le travail de Yanagi visait à déconstruire cette conception moderne de l'art japonais forgée à partir de critères occidentaux - comme la rareté et l'originalité - et à exalter la beauté artisanale de ses productions populaires et anonymes. Il s'inscrivait dans un refus de l'approche biographique et attributionniste de l'histoire de l'art et du culte moderne de l'individualisme ${ }^{5}$. Cette entreprise intellectuelle avait aussi une dimension utopique et sociale, celle de la défense d'un art " rural " - Yanagi qualifia parfois les Ötsu-e de peasant painting ${ }^{6}$-, contre celui des élites urbaines, et elle visait à proposer un nouveau " canon esthétique ${ }^{7}$ ". On ne saurait cependant voir dans ces revendications, comme l'ont pensé un peu rapidement certains historiens ${ }^{8}$, une simple forme de nationalisme culturel issu de l'idéologie agrariste de Meiji, et encore moins une communauté d'esprit avec l'idéal esthétique fasciste d'avant-guerre, comme l'ont soutenu des études produites aux États-Unis dans les années $2000^{\circ}$, même s'il faut reconnaître une certaine convergence au début des années 1940 entre la politique de valorisation des cultures locales par l'État japonais dans un contexte de guerre, et des projets du mouvement Mingei de soutien à l'artisanat, notamment dans les régions pauvres du Nord-Est perçues comme le conservatoire d'un mode de vie rural et de productions marquées par la simplicité ${ }^{10}$. 


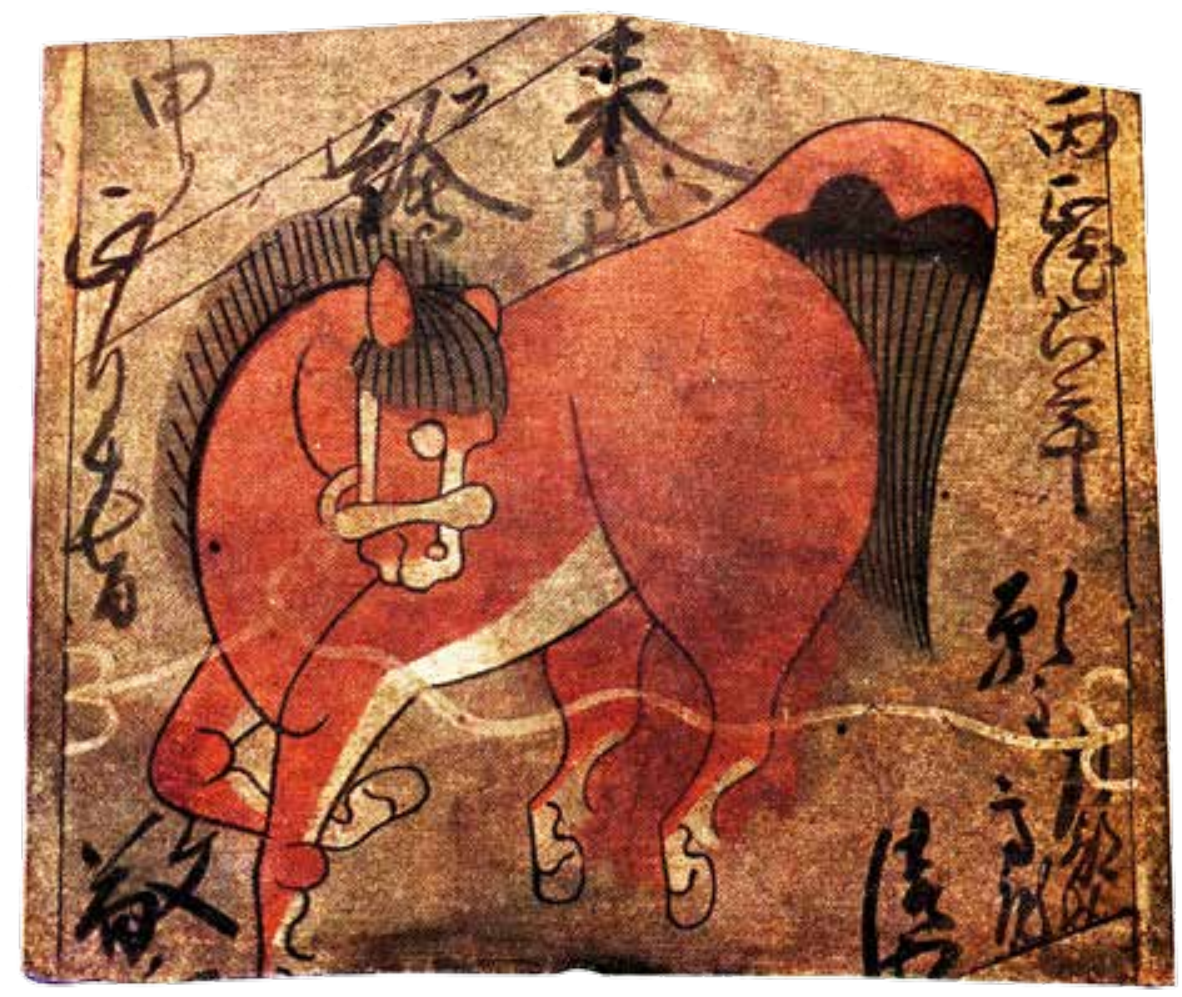

On observe au contraire, dans le projet initial de Yanagi, dans les deux décennies qui précèdent, un processus assez différent de la création politique de l' " art populaire "

1. Plaquette votive peinte, Hachinohe, Okada kannon-dō, 1716, d'après Kōgei, $\mathrm{n}^{\circ} 17$, mai 1932. en Europe au XIX ${ }^{e}$ siècle, lequel avait été soutenu par les États-nations comme une construction idéologique à visée nationaliste ${ }^{11}$. À cet égard, le désintérêt du musée impérial de Tōkyō, à la fin années 1920, vis-à-vis de la proposition de Yanagi de faire don de sa collection d'art populaire, est révélateur de la distance idéologique entre le monde officiel des musées et le projet yanagidien, lequel se réalise finalement exclusivement grâce à des fonds privés ${ }^{12}$. En 1936, alors que Yanagi parvenait finalement à ouvrir à Tōkyō son musée des arts populaires (Nihon mingeikan), après dix ans de combat, une tout autre proposition était faite au gouvernement par Shibusawa Keizō (1896-1963), celle de créer un "musée d'ethnologie du Japon " (Nihon minzoku hakubutsukan) sur la base de sa propre collection d'objets de la vie quotidienne, issue de l'Attic Museum mis en place à son domicile dès les années $1920^{13}$. Bien que cette proposition ne fût finalement pas retenue par l'État et resta, elle aussi, une initiative privée, elle montre la grande différence idéologique entre les deux projets : celui de Yanagi, fondé sur une vision avant tout esthétique, et celui de Shibusawa, dont les collections sont le fruit de recherches ethno-folkloriques et visent implicitement à mettre en avant l'identité culturelle du Japon ${ }^{14}$.

Il est intéressant de constater que les deux musées comprenaient néanmoins certains artefacts de même nature. Il en est ainsi des plaquettes votives peintes (ema; fig. $\mathbf{1}$ ), un type d'objet que Yanagi et les membres du mouvement Mingei collectionnèrent avec ferveur, bien que pour des raisons différentes de celles de Shibusawa, non pas ethnologiques, mais esthétiques, car elles relevaient d'une peinture populaire " naïve, sereine et naturelle ", qui laisse à celui qui l'observe la capacité de " développer son imaginaire ${ }^{15}$ ». Pour Yanagi, la beauté de ces peintures de peu de valeur et considérées parfois comme "infantiles ", 
vient du respect de règles formelles que seuls des "peintres-paysans " sont capables de reproduire par leurs gestes ancestraux, car ils ne sont pas engagés comme les artistes dans une logique de création ego-centrée.

Entre 1929 et 1960, dates de la publication de ses deux grands ouvrages consacrés aux peintures populaires de la région d'Ôtsu ${ }^{16}$, Yanagi n'a eu de cesse de collectionner ${ }^{17}$, de préserver et de défendre cette " autre esthétique " picturale, qu'il reliait avant tout à une tradition artisanale plutôt qu'à une tradition artistique ${ }^{18}$. En ce sens, Yanagi partagea avec William Morris (1834-1896), initiateur du mouvement Arts and Crafts, le rejet de l'industrialisme et la défense d'une tradition artisanale. Mais contrairement à ce dernier, qui dans sa démarche écartait la peinture des arts appliqués à cause de son absence d'utilité en dehors d'une ouvre architecturale ${ }^{19}$, Yanagi la considéra comme un art manuel à part entière, possédant une vocation utilitaire.

Les critères essentiels qui caractérisent pour Yanagi cette peinture populaire et la distinguent de la peinture relevant des " beaux-arts " étaient initialement au nombre de trois : anonymat (absence de signature), multiplicité et modicité ${ }^{20}$. Il y ajouta par la suite un ensemble de caractéristiques, comme le caractère traditionnel des motifs, la rapidité d'exécution, la réduction du motif à l'essentiel, le caractère a-personnel, la facture artisanale et non artistique, ou encore l'expression de la beauté issue de la "force tierce " (tariki), concept emprunté au bouddhisme amidiste ${ }^{21}$.

2. Première de couverture du catalogue de l'exposition Peintures d'Ōtsu : regards européens, Ōtsu City Museum of History, 2019.

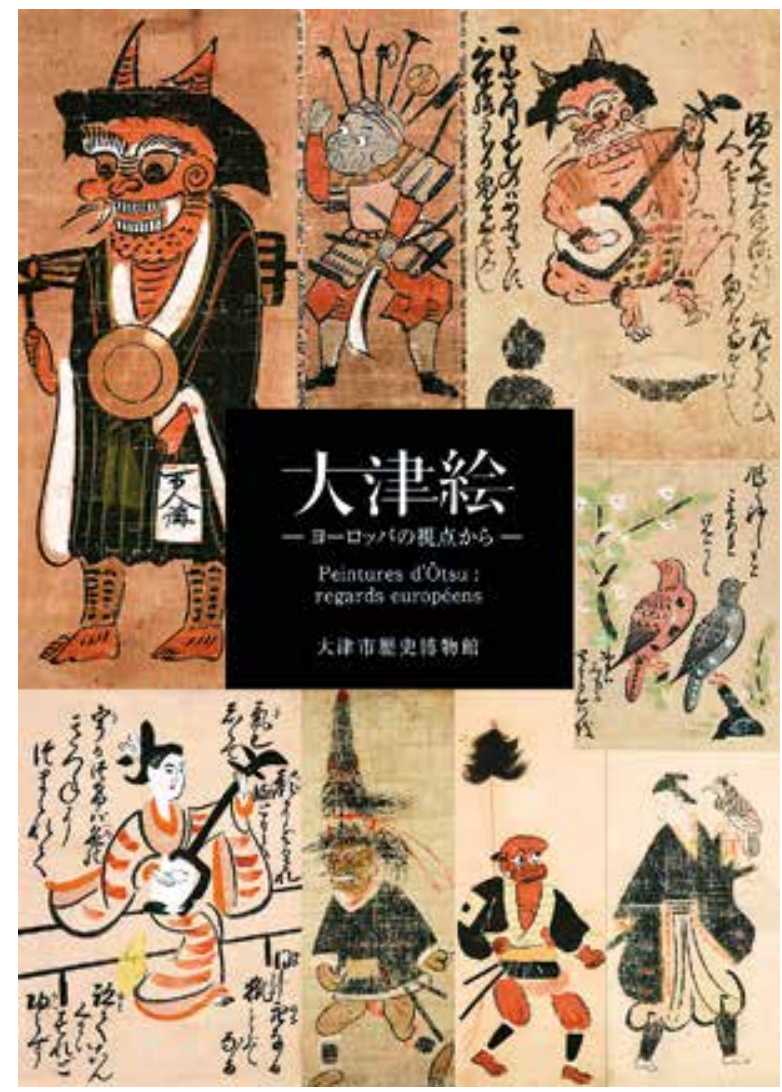

Le genre pictural que Yanagi identifia comme correspondant le mieux à son projet de valorisation d'une "peinture artisanale " fut l'imagerie d'Ōtsu' ${ }^{22}$ (fig. 2), une imagerie apparue au début du XVII siècle entre Kyōto et le relais d'Ōtsu, originellement à thème religieux, bouddhique ou shintō, mais aussi liée à des rites d'origine taoïste pratiqués dans le monde rural, qui évolua ensuite vers une forme profane et didactique. Son caractère " primitif " venait principalement de la modestie de ses moyens techniques et de ses contraintes économiques de production - rapidité d'exécution à l'aide de pochoirs, limitation de la palette, simplification à l'extrême du motif - mais aussi de sa nature populaire et de sa fonction utilitaire.

Pour Yanagi, la qualité principale de ces images d'Ōtsu repose, comme pour les peintures votives, sur leur stéréotypie formelle et sur la sûreté du geste des artisans-peintres qui les ont produites. Mais il ne les considérait pas uniquement comme des peintures de terroir, admirables par leur simplicité. Il voulut y voir dans certains cas, lorsqu'elles prirent une forme plus caricaturale, un acte de résistance à l'autorité, dans le contexte social et politique de l'époque d'Edo où toute 
prise de parole critique était exclue pour les gens du peuple. D'où son refus de parler, au point de vue de son contenu, de "peinture primaire " (genshi-teki kaiga) : elle témoignait en effet de l' " esprit " du petit peuple et elle était selon lui " l'unique moyen à cette époque pour les gens du commun de critiquer la société ", par le recours à l'allusion ou à l'humour ${ }^{23}$. L'écrivain Nagai Kafū partagea avec Yanagi cette interprétation de certains genres de l'art d'Edo comme des formes de résistance populaire par la veine du divertissement ${ }^{24}$. S'il récuse, pour les raisons que nous venons de voir, le terme de "primitives " pour qualifier les peintures d'Ōtsu en général, Yanagi insiste sur la qualité artistique de celles de la toute première période au XVII ${ }^{\mathrm{e}}$ siècle, considérant que celles qui suivirent ne furent souvent que les pâles et décadentes copies des plus " archaïques ". C'est ce questionnement sur le sens de cette imagerie et de son prétendu primitivisme qui a été au centre du colloque « Décrypter l'imagerie populaire d'Ōtsu : des imagiers de l'époque d'Edo à Miró ", organisé en 2016 à la Maison franco-japonaise, et dont les actes ont paru dans un numéro spécial de la revue Bijutsu fōramu 21, sous le titre « Primitives Pictures? Ötsu-e Alive in the Modern World ${ }^{25} "$.

Les années 1920 furent aussi un moment de redécouverte d'autres formes "primitives " de la peinture d'Edo, qui eurent désormais droit à leur histoire, grâce aux publications de deux maîtres de la peinture moderne, Kishida Ryūsei (1891-1929) et Yoshikawa Kanpō (1894-1979), consacrées respectivement aux peintures $u$ kiyo-e de la première période ${ }^{26}$ et aux représentations de créatures surnaturelles (yōkai, oni, bakemono, yürei, etc.), tant appréciées aujourd'hui par le grand public ${ }^{27}$. À la même époque, l'histoire du dessin caricatural - envisagée depuis les rouleaux satiriques du XII e siècle sur les animaux, jusqu'aux dessins de presse du début du XX siècle -, fit elle aussi l'objet d'une toute première compilation, par le dessinateur de manga Hosokibara Seiki (1885-1958) ${ }^{28}$. On notera que les peintures d'Ōtsu, en cours de réhabilitation par Yanagi, trouvèrent déjà, par leur dimension primitive et satirique, leur place dans ces trois publications pionnières qui mettaient en avant des formes marginalisées de la peinture populaire prémoderne. Dans ces mêmes années 1920, un dessinateur comme Okamoto Ippei (1886-1948) réinterpréta les images d'Ōtsu dans des dessins politiques publiés à la une de la presse quotidienne, preuve que leur aspect satirique était une évidence.

Le corpus pictural défini par Yanagi est maintenu dans la plupart des ouvrages et des catalogues d'exposition consacrés à l'art populaire, au Japon comme en Occident. Hugo Munsterberg (1916-1995), historien de l'art asiatique formé à Harvard, qui vécut au Japon dans la première moitié des années 1950, fut un proche de Yanagi et de son mouvement. Dans The Folk Arts of Japan (1958), premier ouvrage de synthèse en anglais sur le Mingei, préfacé par ce dernier, un chapitre est consacré à la peinture et à la sculpture. Munsterberg insiste sur l'« archaïsme " de la folk painting de l'époque d'Edo, qui en fait l'attrait pour un oeil moderne et il y voit la "version primitive " de la grande peinture des siècles antérieurs $^{29}$. La révélation de l'art populaire par le mouvement Mingei est analysée comme un prolongement de l'intérêt pour les « les civilisations et les arts primitifs » qui parcourt l'art moderne depuis Gauguin, ou du nouveau regard porté sur les dessins d'enfants, grâce à l'appréciation des œuvres de Rousseau ou de $\mathrm{Klee}^{30}$. En quelque sorte, ce serait le filtre de l'art moderne qui aurait rendu possible cette redécouverte, de même que ce serait l'industrialisation et l'urbanisation de la société qui auraient poussé à un retour nostalgique vers un art populaire des villages, synonyme de fraîcheur et de simplicité, délivrant de la sophistication et d'une conscience artistique trop prégnante.

On ne saurait oublier qu'au Japon même, la redécouverte de ces peintures par Yanagi, mais aussi par des peintres modernes, ne fut possible que grâce à une sorte de dépaysement du regard qui passa par l'art moderne occidental ${ }^{31}$. L'appréciation 
de ces peintures populaires par Yanagi n'est pas étrangère à son goût pour l'art médiéval et William Blake, ou à son penchant pour les peintres postimpressionnistes, notamment Van Gogh et Gauguin, vis-à-vis desquels il développa, avec les membres de la revue littéraire Shirakaba ${ }^{32}$ qui les introduisirent au Japon dans les années 1910, une sorte de romantic primitivism, pour reprendre l'expression de Robert Goldwater dans son ouvrage pionnier, Primitivism in Modern Art (1938). Il est intéressant de noter que ce sont les artistes qui s'étaient confrontés à la peinture contemporaine lors de leurs séjours en France, qui firent les plus vibrants éloges de la peinture d'Ōtsu : Umehara Ryūzaburō établit ainsi en 1929 d'audacieuses comparaisons avec le style fauve de Matisse et les œuvres de Rouault, tandis qu'Okamoto Tarō, proche du milieu surréaliste parisien d'avant-guerre, y vit, dans les années 1970, pas moins qu'une source pour revitaliser un art contemporain tombé dans le formalisme ${ }^{33}$.

Cependant, aujourd'hui ce n'est guère cette dimension qui est mise en avant lorsque l'on évoque la peinture populaire de l'époque d'Edo ou le Mingei. C'est plutôt la promotion par Yanagi d'une création contemporaine puisant aux sources du Mingei qui est privilégiée, comme en témoigne la récente exposition qui eut lieu au musée du design à Tōkyō - MINGEI: Another Kind of Art (21_21 Design Sight, 2018) -, organisée par le célèbre designer Fukasawa Naoto (né en 1956), actuel directeur du Japan Folk Crafts Museum de Tōkyō fondé par Yanagi en 1936. Il est révélateur que la peinture n'y était pas présente, comme elle avait également été absente de l'exposition organisée par Germain Viatte au musée du Quai Branly en 2008, L'esprit Mingei au Japon, de l'artisanat populaire au design, qui se focalisa sur les liens entre le mouvement historique de Yanagi et la création contemporaine, via Charlotte Perriand ou Isamu Noguchi ${ }^{34}$. Le Mingei semble avoir oublié ses sources graphiques pour insister exclusivement sur son rôle d'inspirateur du design d'aujourd'hui ou de précurseur d'un mode de vie et de rapport à l'objet fondé sur la sobriété et le local, qui ressurgit dans le contexte de la société post-Fukushima, comme l'annonce l'anthropologue Nakazawa Shin'ichi dans l'ouvrage La leçon du Mingei : technique de l'imperfection ${ }^{35}$.

Cette perception pourrait néanmoins changer, grâce à l'exposition Mō hitotsu no Edo kaiga: Ōtsu-e /Ōtsu-e: Another History of Edo Painting qui se tient de mai à novembre $2020 \mathrm{au}$ Fukushima Prefectural Museum of Art, puis à la Tōkyō Station Gallery. Cette exposition propose de reconsidérer les images d'Ōtsu, non plus comme des "peintures folkloriques ", mais de revenir sur le regard des artistes japonais - du peintre lettré Tomioka Tessai (1836-1924) au designer textile Serizawa Keisuke (1895-1984) - qui, depuis la fin du XIX ${ }^{\mathrm{e}}$ siècle, les ont redécouvertes et appréciées, fascinés à la fois par leur primitivisme et leur modernité, y puisant pour certains des sources d'inspiration, comme le fit en premier le peintre occidentaliste Asai Chū (1856-1907) à son retour d'un séjour en France au début des années $1900^{36}$.

\section{La peinture d'Edo comme art d'avant-garde : de la redécouverte des années 1970 à Murakami}

Une étape fondamentale dans la reconnaissance de formes picturales non conventionnelles de l'époque d'Edo revient aux travaux du grand historien de la peinture prémoderne Tsuji Nobuo (né en 1932) ${ }^{37}$ et à la publication de Kisō no keifu [Lignage des excentriques] $^{38}$ (fig. 3) en 1970, qui fut considéré aussitôt comme un "défi à l'histoire de l'art $\operatorname{orthodoxe}^{39}$ ". Dans cet ouvrage qui fit date, dont le sous-titre était, lors de sa parution 
en revue, « l'avant-garde à l'époque d'Edo ", l'auteur envisage une sorte de filiation entre les ouvres de plusieurs maîtres singuliers et oubliés de l'époque d'Edo, depuis Iwasa Matabei et Kanō Sansetsu au début du XVII siècle, jusqu'à Kuniyoshi, le dernier grand artiste de l'ukiyo-e dans le premier XIX siècle, en passant par Itō Jakuchū, Soga Shōhaku et Nagasawa Rosetsu, actifs à Kyōto dans la seconde moitié du XVIII ${ }^{\mathrm{e}}$ siècle. J'ai eu la chance, au tout début des années 1990, de bénéficier de l'enseignement de Tsuji Nobuo à l'université de Tōkyō et de participer à des voyages d'étude en sa compagnie, et j'ai pu mesurer la nouveauté de son approche de la peinture de l'époque d'Edo, qui allait à l'encontre d'une description linéaire des courants majeurs, comme les écoles Kanō et Rinpa, le naturalisme de Maruyama Ōkyo, l'école des lettrés ou l'ukiyo-e. Ses travaux ont permis de faire émerger dans le champ de l'histoire de l'art certaines figures perçues comme des personnalités indépendantes, dont les productions atypiques se distinguent des styles de ces grandes écoles de peinture, et de produire une vision plus organique du développement de l'art de cette période. Ses travaux ont conduit également à réévaluer leur œuvre selon une sensibilité et des valeurs esthétiques contemporaines, réévaluation qui permet à Tsuji de qualifier Jakuchū de "primitif » et même de "surréaliste ${ }^{40}$ ". L'époque d'Edo apparut dès lors comme un moment unique dans l'histoire de la peinture japonaise, où émergèrent des personnalités artistiques hors du commun, qualifiées par Tsuji de kisō - littéralement des peintres à la " pensée originale »-, terme positif emprunté à l'expression kisō tengai, qui insiste sur la singularité ou l'audace d'une idée qui " tombe du ciel ", mais que l'on rend à défaut par " excentrique ». Leur œuvre se caractérise principalement par une grande liberté de style, en rupture avec les canons académiques, et une forme d'innovation. Cette nouvelle perception de la peinture d'Edo s'est finalement imposée, comme en témoigne la récente publication d'une collection complète sur l'art japonais, dans laquelle la peinture de cette période occupe une place beaucoup plus importante et intègre ces artistes qui étaient à la marge, bien qu'ils fussent appréciés de leur temps ${ }^{41}$.

Le cas du moine Hakuin Ekaku (1685-1768), rénovateur du zen Rinzai dans la première moitié du XVIII ${ }^{e}$ siècle, qui utilisa la peinture comme un support pour diffuser la foi auprès des paysans lors de ses pérégrinations à travers tout le Japon, est emblématique de ces " extravagants ", dont la puissance de l'œuvre nous frappe malgré leur apparente maladresse technique (fig. 4). Dans un ouvrage publié vingt ans plus tard, Kisō no zufu [Album des excentriques] / Curiosity in Japanese Art (1989), Tsuji approfondit son approche et consacra toute une partie à la " puissance créatrice de l'amateurisme ", en réunissant Jakuchū, Sharaku et Hakuin. Les œuvres de ce dernier fascinent, pour Tsuji, par " la force de l'inhabileté " (chisetsu no hakuryoku) qu'elles dégagent. Si le terme d'amateur ne saurait s'appliquer avec le même sens à Jakuchū qu'aux
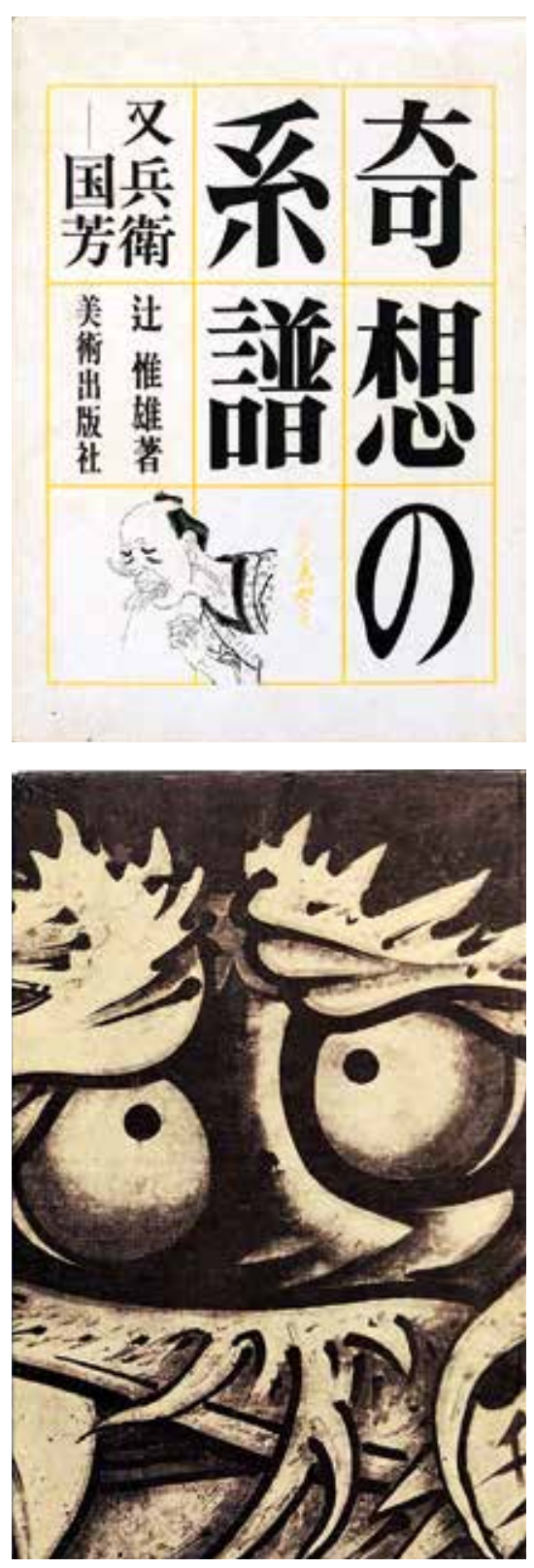

3. Coffret du livre de Tsuji Nobuo, Kisō no keifu, 1970, avec un détail de Soga Shōhaku, Dragon dans les nuages, 1763, Boston, Museum of Fine Arts. 


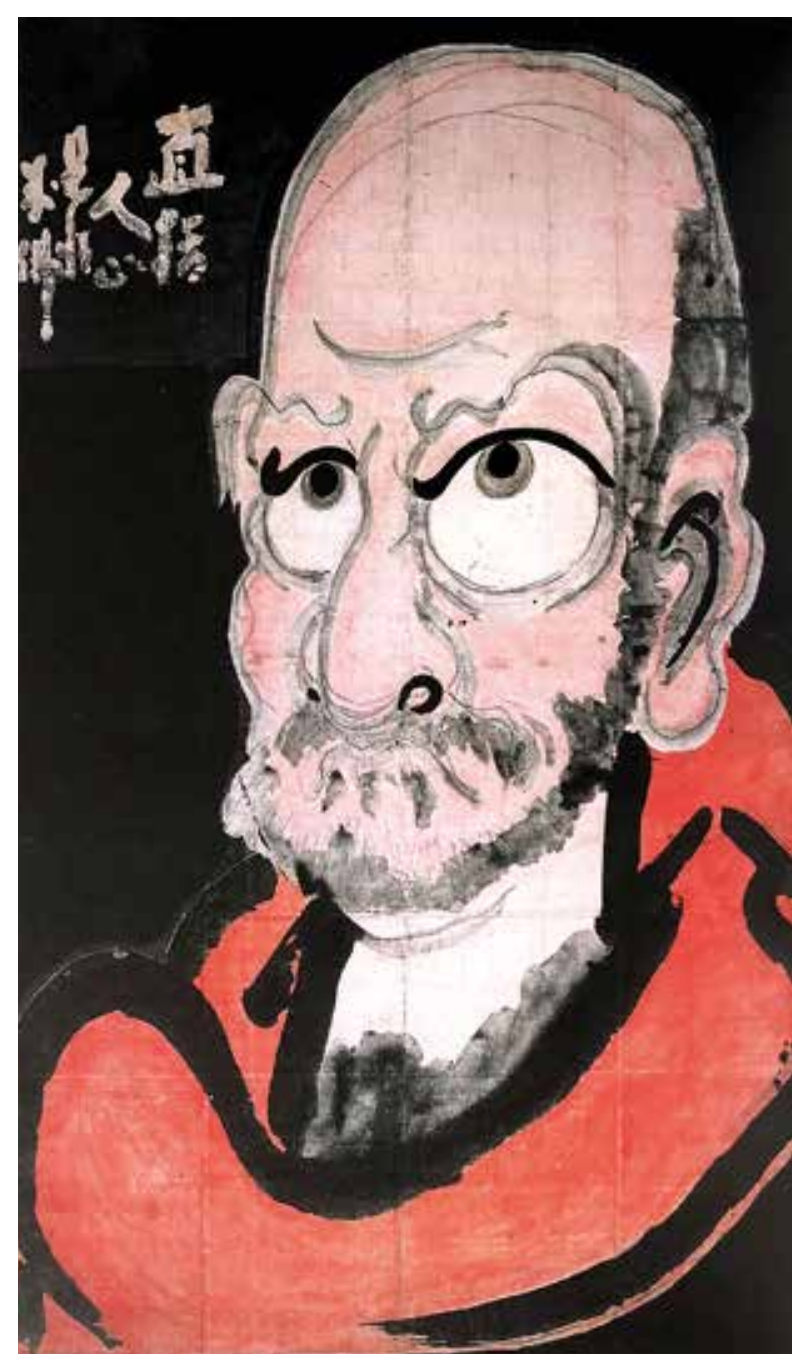

4. Hakuin, Bodhidharma, vers 1767 , encre et couleurs sur papier, $192 \times 112,5$ cm, Ōita, monastère Manju-ji, d'après Tsuji Nobuo, Kisō no zufu, 1989. deux autres, l'absence de perfection technique, la déformation volontaire du motif et la recherche de la puissance de l'effet graphique sont bien des caractéristiques communes à l'art de Hakuin et de Sharaku.

Un demi-siècle après la publication du premier ouvrage de Tsuji, ces artistes qui étaient à la marge de l'histoire de l'art sont devenus pour la plupart des "stars " des expositions temporaires, au point de bouleverser notre vision de l'art d'Edo. En 2019, l'exposition Kisō no keifu: Edo kaiga mirakuru wārudo / Lineage of Eccentrics: The Miraculous World of Edo Painting (Tōkyō Metropolitan Museum of Art), qui reprenait tel quel le titre de l'ouvrage de Tsuji, présentait huit de ces peintres du XVII au XIX ${ }^{e}$ siècle, marque de leur consécration auprès du grand public et de ce renversement de valeurs.

Cette reconnaissance des excentriques a trouvé un prolongement inattendu avec le dialogue créatif poursuivit entre 2009 et 2011 entre Tsuji Nobuo et l'artiste contemporain Murakami Takashi (né en 1962). Le premier inspirant au second une série de vingt et une créations, sur la base de ses essais sur la peinture japonaise, selon le principe des traditionnels " concours de peinture " (e-awase) qui consistaient à confronter deux ouvres et à en débattre des mérites respectifs. Murakami a expliqué la révélation que fut pour lui la lecture du travail de Tsuji, et son influence décisive sur son œuvre, en le poussant à réexaminer sa pratique artistique. Cela l'a convaincu de la possibilité de produire une peinture contemporaine qui ne soit pas marquée par un "suivisme " de l'art occidental - une tendance lourde selon lui chez les artistes japonais depuis Meiji - en puisant ses sources chez les excentriques de l'époque d'Edo en particulier, auxquels il rend des hommages souvent parodiques. Ces " joutes " autour de l'histoire de l'art japonais revisité par Tsuji furent réunies en $2014^{42}$, au moment où ce dialogue entre le peintre et l'historien de l'art fut mis en scène dans l'exposition de Murakami autour de sa réinterprétation, en une immense fresque de cent mètres de long, des surprenantes peintures de cinq cents arhat que Kanō Kazunobu réalisa au milieu du XIX siècle (Murakami Takashi no Gohyaku rakan-zu, Mori Art Museum, 2015). L'expérience fut prolongée en 2017 par l'intéressante mise en résonnance du travail de Murakami avec le regard de Tsuji et les collections du musée de Boston, dans l'exposition Takashi Murakami: Lineage of Eccentrics ${ }^{43}$. Les collections japonaises de ce musée sont initialement issues de la collecte menée au Japon dans 
le dernier quart du XIX siècle par Ernest Fenollosa et Okakura Tenshin, lorsqu'ils forgèrent une histoire canonique de l'art japonais, en mettant en avant sa tradition religieuse et aristocratique, fondée sur l'académisme des grandes écoles de peinture. Mais il s'y trouve aussi plusieurs chefs-d'œuvre " excentriques » de Jakuchū et de Shōhaku, rapportés principalement par William Sturgis Bigelow, comme le Dragon dans les nuages (1763), qui orne le coffret du livre de Tsuji (fig. 3) et fut réinterprété par Murakami dans une composition monumentale de dix-huit mètres de long, en monochrome rouge, sous le titre Dragon in Clouds - Red Mutation (2010).

À l'inverse de l'approche de Yanagi, qui privilégiait la peinture populaire d'Edo à caractère primitif, mais fondamentalement anonyme, Tsuji s'intéressa à des artistes remarquables tant par la singularité de leur personnalité que par l'originalité de leur œuvre, mais qui étaient relégués à la " marge ". Ces deux approches combinées ont ouvert des perspectives radicalement neuves, permettant une relecture de l'art d'Edo selon une sensibilité contemporaine, dont témoignent de nombreuses expositions organisées ces quinze dernières années, au point que l'on a pu parler d'un véritable « boom de la peinture d'Edo ».

\section{Pour une autre peinture d'Edo : le tournant des années 2000}

Depuis la fin des années 2000 se sont multipliées les expositions sur d'autres dimensions méconnues de la peinture d'Edo, appréciées du grand public essentiellement pour leur naïveté, leur légèreté innocente ou leur humour parodique, témoignant d'une évolution du regard sur le primitivisme des arts de la période prémoderne. Certaines ont connu des extensions en France, comme Nihon bijutsu ga warau / The Smile in Japanese Art (Tōkyō, Mori Art Museum, 2007), présentée sous le titre Warai : l'humour dans l'art japonais. De la préhistoire au XIX $X^{e}$ siècle (Paris, Maison de la culture du Japon à Paris, 2012), qui faisait la part belle aux images d'Ōtsu révélées par Yanagi, mais aussi à ces artistes « excentriques " d'Edo redécouverts par Tsuji, comme Shōhaku, Hakuin ou Kuniyoshi.

Si la période d'Edo n'est pas la seule concernée par ces expositions, dont certaines balaient l'art japonais depuis le Jōmon jusqu'au $\mathrm{XX}^{\mathrm{e}}$ siècle, elle constitue néanmoins le moment privilégié de l'émergence d'une peinture non-professionnelle, primitive au sens de brute ou de maladroite, d'expression " naïve " et souvent sous-tendue par l'humour. Ces expositions revisitent l'histoire de la peinture à travers des dénominateurs communs formulés par un adjectif ou un motclé qui sert de fil rouge, comme kawaii ( "adorable ${ }^{44}$ "), soboku ( " primitif ${ }^{45}$ ") ou encore hokkori ( "apaisant ${ }^{46}$ "). Elles proposent une approche non historicisée de l'art japonais, qui brise les cadres chronologiques, en se fondant sur l'appréciation immédiate des œuvres dans leur dimension sensible et en faisant découvrir un ensemble de peintures qui n'appartiennent pas au corpus établi par l'histoire de l'art académique. Une dizaine d'expositions majeures ont été organisées dans cette optique depuis 2007. Nous n'en présenterons

5. Première de couverture du catalogue de l'exposition Hesomagari Nihon bijutsu, Fuchū Art Musuem, 2019, avec un détail de Tokugawa lemitsu, Lièvre, première moitié du XVII ${ }^{\mathrm{e}}$ siècle, Japon, collection privée.

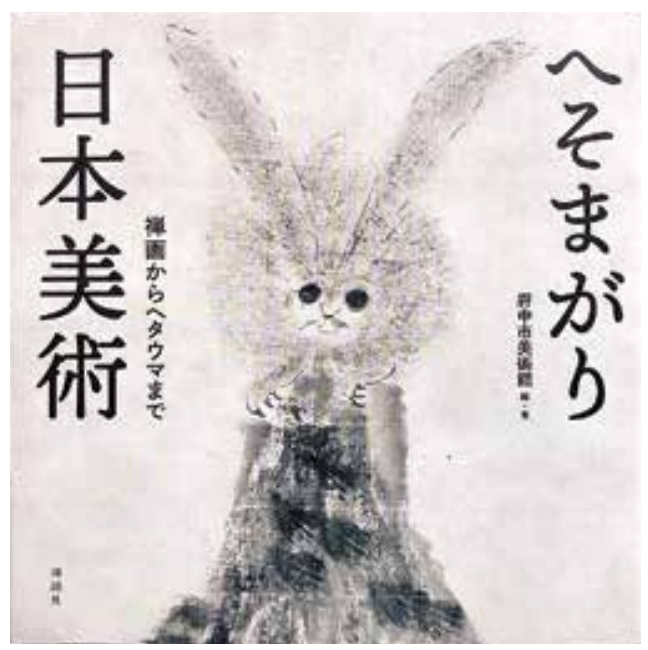


que les deux plus récentes, qui se sont tenues à Tōkyō l'année dernière. L'exposition Hesomagari Nihon bijutsu. Zen kara heta-uma made [Un art japonais tortueux. De la peinture zen à la gaucherie touchante] (Tōkyō, Fuchū Art Museum, 2019, fig. 5) présente cent trente-huit ouvres allant des puissants lavis d'encre du moine Sesson au XVI ${ }^{\mathrm{e}}$ siècle, jusqu'au peintre avant-gardiste Yorozu Testugorō (1885-1927), marqué par le fauvisme et le cubisme, et qui réinventa dans les années 1910 une forme singulière de peinture dans le style dilettante des lettrés. Si le déroulé suit la chronologie historique, le vrai fil d'Ariane de l'exposition est la mise en avant du heta-uma (littéralement la " gaucherie réussie ") : des œuvres imparfaites au regard du canon esthétique classique, mais qui touchent notre sensibilité par leur fraîcheur, leur puissance primitive. La couverture du catalogue reproduit ainsi une curieuse peinture d'amateur, représentant un lièvre de face, réalisée par le shōgun Tokugawa Iemitsu (1604-1651) et qui a été découverte récemment. Ce charme indicible de la maladresse technique était déjà exprimé à l'époque d'Edo dans de brefs poèmes, comme celui qui demande : «Les peintures d'Ōtsu / Sont-elles habiles ou maladroites ? / On ne saurait le dire. " (Chikushi, 1818).

La seconde exposition, Nihon no soboku-e. Yurui, kawaii, tanoshii bijutsu [La peinture primitive au Japon. Un art décomplexé, adorable et ludique] (Tōkyō, Mitsui Memorial Museum, 2019, fig. 6) s'attache également à faire découvrir dans la peinture japonaise depuis les premiers rouleaux bouddhiques du vIII siècle jusqu'aux croquis minimalistes de Keisai à la fin du XVIII ${ }^{e}$ siècle -, une forme de beauté primitive. Le mot-clé est soboku ( " primitif "), auquel la traduction à connotation négative de "naïf " ne rend pas justice. Ce terme renvoie plutôt à une chose laissée dans son état naturel, à une forme de simplicité considérée comme une qualité. Si l'expression n'avait pas pris un autre sens, nous pourrions parler $\mathrm{d}^{\prime}$ " art brut ».

Les historiens de l'art Kaneko Nobuhisa

6. Première de couverture du catalogue de l'exposition Nihon no soboku-e, Mitsui Memorial Museum, 2019.

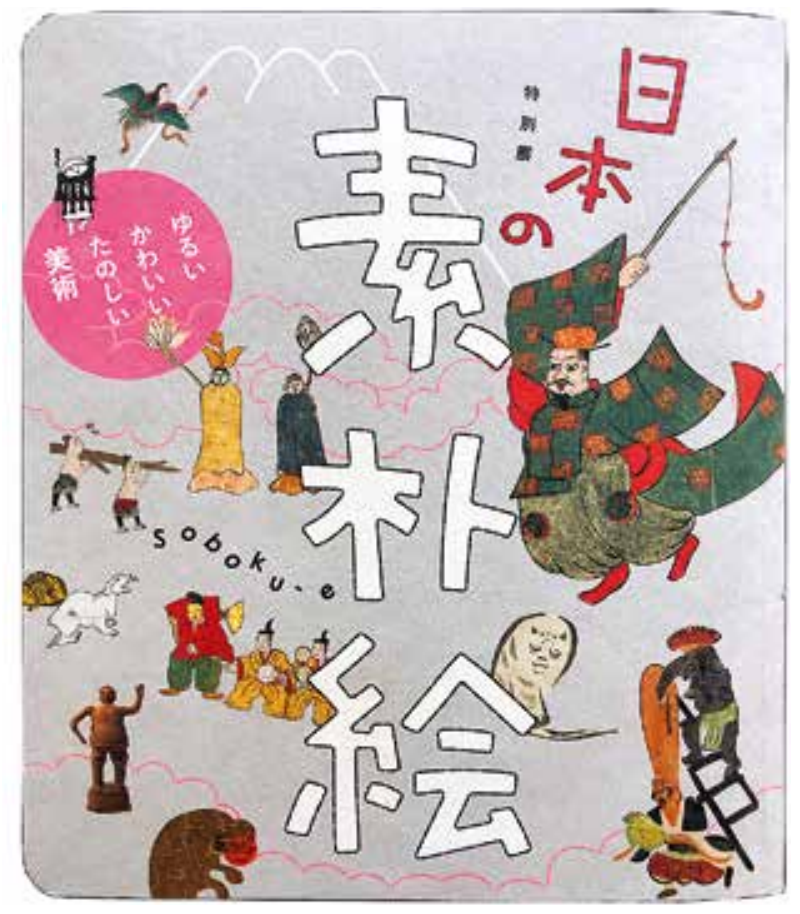
(né en 1962) et Yajima Arata (né en 1960), respectivement commissaires de la première et de la seconde exposition, sont les acteurs de la redécouverte et de la valorisation de ce primitivisme de la peinture japonaise. Yajima a été longtemps conservateur au musée Shōtō de Shibuya à Tōkyō, où il organisa en 1993 sa première exposition, consacrée à la peinture liée aux croyances populaires au Moyen Âge, suivie d'un très grand nombre d'expositions qui portèrent un regard novateur sur les arts populaires de la période d'Edo. Ses travaux touchent notamment aux formes locales et marginales d'art bouddhique populaire produites par des artistes non-professionnels, comme les sculptures de Mokujiki, les peintures des moines zen Hakuin et Sengai, les peintures des enfers ou encore les peintures votives ${ }^{47}$. Kaneko, conservateur au musée de Fuchū, ouvert en 2000 dans la banlieue ouest de Tōkyō, a lui aussi multiplié ces dernières années les expositions temporaires sur la 


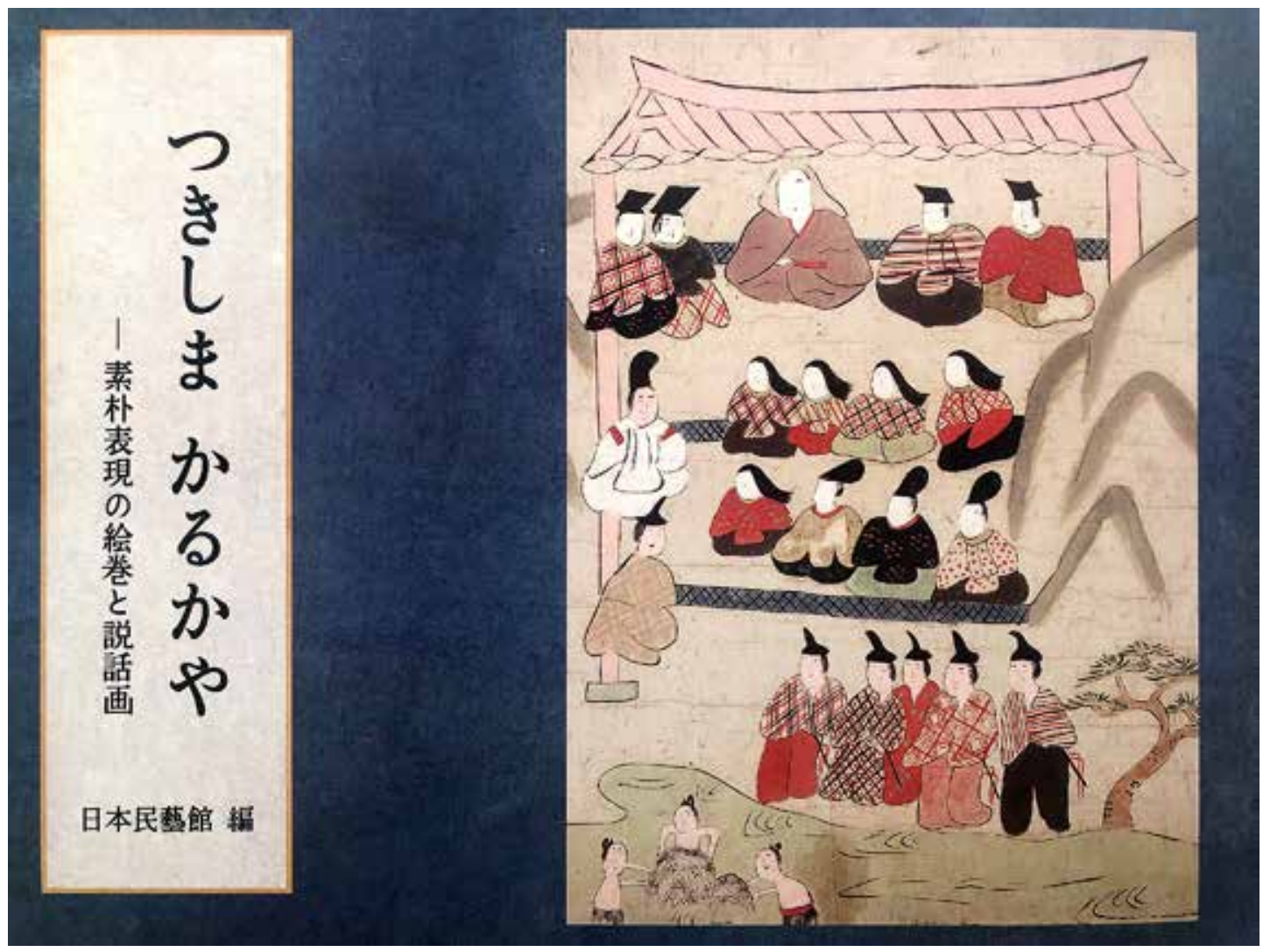

peinture d'Edo selon un point de vue original, et s'est 7. Première de couverture du catalogue de l'exposition Tsukishima, Karukaya, The Japan Folk Crafts Museum, 2013, avec un détail du Rouleau du conte de l'île artificielle, $\mathrm{xVI}^{\mathrm{e}}$ siècle, The Japan Folk Crafts Museum.

Parallèlement aux nombreuses expositions dont ils ont été commissaires, Yajima et Kaneko ont fait paraître de nouvelles histoires de l'art japonais qui s'écartent des chefs-d'œuvre reconnus et des œuvres " grandioses ", pour s'attacher à décrypter une autre sensibilité populaire qui parcourt l'art japonais. Yajima a ainsi publié Nihon no soboku-e [La peinture primitive au Japon] (Tōkyō, Pie Books, 2011) et Yurukawa Nihon bijutsu-shi [Histoire d'un art japonais décomplexé et adorable] (Tōkyō, Shōdensha shinsho, 2019), deux ouvrages aux argumentaires particulièrement convaincants qui marquent une nouvelle étape dans la redécouverte de tout un pan enfoui de la production picturale du Japon. Kaneko a fait paraître Nihon otoboke kaiga-shi. Tanoshii Nihon bijutsu [Histoire de la peinture japonaise faussement naïve. L'art japonais ludique] (Tōkyō, Kōdansha, 2016) qui vise plutôt à valoriser tout un courant grotesque et humoristique qui parcourt la peinture japonaise, depuis le Moyen Âge jusqu'aux années 1920.

Cette nouvelle sensibilité à la dimension primitive de la peinture populaire prémoderne a conduit le Japan Folk Crafts Museum à organiser en 2013 une exposition sur l' « expression naïve » dans la peinture narrative à thème bouddhique de l'époque d'Edo, à partir de deux rouleaux du XVI e siècle : le Conte de l'île artificielle (fig. 7) et Le Saint homme Karukaya (Tōkyō, Suntory Museum of Art), deux contes édifiants illustrés qui sont parmi les plus anciens de 


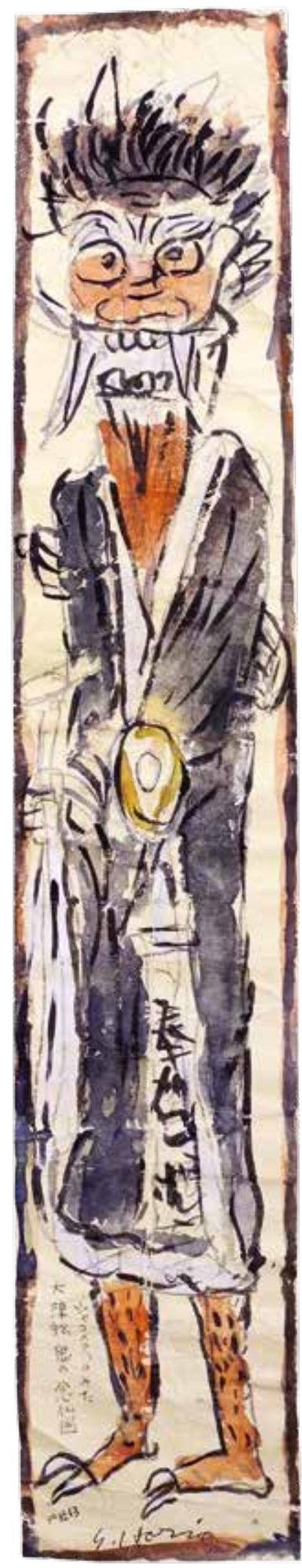

ce genre qui nous soient parvenus ${ }^{48}$. Yanagi avait découvert la première de ces ouvres dès 1935 et l'avait acquise pour le Nihon mingeikan (Japan Folk Crafts Museum) ${ }^{49}$, défendant à travers elle une forme de " beauté instinctive " malgré l' « illogisme » de l'expression, une beauté dénuée de technique, née d'un pinceau amateur, mais marquée par une sincérité que ne peuvent atteindre les peintres de métier. Ces deux œuvres étaient confrontées dans l'exposition à une série de peintures anonymes à caractère naïf des XVI ${ }^{e}$ et XVII ${ }^{\text {e }}$ siècles, y compris les plus anciennes peintures d'Ōtsu de la collection de ce musée, des peintures des enfers, des mandalas de pèlerinage et les premières formes de livres xylographiques illustrés. Elles rappelaient le rôle pionnier joué un siècle plus tôt par Yanagi et le mouvement Mingei dans la reconnaissance du primitivisme pictural, qui est de nouveau apprécié aujourd'hui.

L'ethnologue Jean Cuisenier, qui fut directeur du musée national des arts et traditions populaires, a bien souligné les écueils de ce genre de regard contemporain sur l'art populaire, qualifié trop rapidement de " naïf ", lorsqu'il écrit que " rien ne s'oppose tant à une exacte évaluation de l'art populaire que les jugements courants, qui tantôt valorisent les œuvres comme si elles manifestaient la naïveté de leurs auteurs et tantôt y trouvent le charme de l'archaïsme " ${ }^{50}$. D'un strict point de vue d'histoire de l'art également, une telle confrontation d'œuvres produites dans des contextes radicalement différents et aux intentions divergentes peut surprendre, mais elle est conforme au projet yanagidien d'une relecture et d'une mise en valeur des arts graphiques du Japon selon les critères esthétiques du Mingei. C'est à la fois l'originalité et la limite de cet exercice. Yanagi lui-même ne cachait pas que son propos n'était pas celui d'un historien d'art, mais celui d'un philosophe, d'un esthète portant un regard novateur, mais personnel, sur des productions jusque-là ignorées ou négligées par l'histoire de l'art japonais ${ }^{51}$.

\section{En guise de conclusion : Horio, un dialogue contemporain avec le primitivisme}

Cette quête du primitivisme, ce goût pour l'excentrisme ou encore cette revendication de l'humour et de la parodie qui parcourent certains courants de la peinture d'Edo, redécouverts à partir des années 1920, trouvent aussi de nombreux échos dans l'art contemporain, d'Okamoto Tarō à Yamamoto Tarō, en passant par Murakami Takashi.

Le cas de Horio Sadaharu (1939-2018) peut être examiné également dans cette perspective. Artiste autodidacte qui débuta au sein du mouvement avant-gardiste Gutai au milieu des années 1960, il inscrivit la performance au cœur de sa démarche, tout en portant un regard singulier sur les croyances

8. Horio Sadaharu, Peinture d'Ōtsu. Démon invoquant le bouddha Amida, vu par Giacometti, s. d., Paris, collection privée. populaires du Japon ancien. Horio - grand lecteur des travaux du folkloriste Miyamoto Tsuneichi (1907-1981) sur la 
vie rurale ${ }^{52}$-, dialogua ainsi avec des formes primitives de la peinture japonaise, comme dans son démon invoquant le bouddha Amida, inspiré des peintures d'Ōtsu, mais revisité avec humour dans le style des sculptures filiformes de Giacometti (fig. 8).

Horio fut fasciné autant par Tàpies - dont il admirait le travail sur la matière à partir de matériaux pauvres - que par l'esthétique minimaliste du Mingei, découverte notamment grâce à son oncle Horio Mikio (1911-2005), qui était un des soutiens de ce mouvement et grand collectionneur de céramiques de Hamada Shōji, caractérisées par leurs couvertes tachistes réalisées à l'aide de coulures ${ }^{53}$. Il rendit un hommage aux simples dévots de la Véritable école de la Terre pure (Jōdo shinshū), dans une impressionnante série de cent cinquante gravures monochromes monumentales intitulée Biographies d'hommes d'excellence sublimes (Myōkōnin-den, 1992-2009) ${ }^{54}$, en référence au recueil éponyme du milieu du XIX ${ }^{e}$ siècle dédié à ces fidèles du bouddhisme amidiste, souvent d'extraction paysanne. Cet intérêt pour l'école de la Terre pure, fondé sur le principe du Salut par la dévotion inconditionnelle en Amida, fut sans doute suscité par les écrits sur ces hommes pieux laissés par Yanagii ${ }^{55}$, qui avait établi très tôt un parallèle entre la démarche créatrice du Mingei - fondée sur le renoncement à la reconnaissance individuelle au profit de la « force tierce " $(\text { tariki })^{56}-$, et l'attitude des croyants amidistes.

Dans une de ses toutes dernières installations, Art Vending Machine, présentée par la galerie Axel Vervoordt lors de plusieurs foires internationales (Art Brussels, 2015 ; Armory Show, New York, 2017), Horio proposait aux visiteurs, contre un euro ou un dollar symbolique, une ouvre " originale " peinte à l'encre en moins d'une minute, parmi un choix de dix sujets : peinture sonore, moqueuse, instantanée, à la Sada[haru], etc. Ce distributeur automatique à l'esprit dadaïste, produisant des œuvres à la chaîne - dont des imitations de Picasso ! - interrogeait non seulement avec humour la valeur de l'œuvre d'art, mais il se voulait peut-être aussi l'application des principes du Mingei énoncés par Yanagi, comme la répétition du geste artisanal et le refus de la subjectivité, de l'ego, dans la démarche artistique. Par-delà l'ironie, Horio - qui n'avait jamais voulu devenir un artiste " professionnel " pour se garantir une totale liberté de création - réhabilitait l'esprit de la fast painting réalisée à la demande devant le client de passage, par des artisans anonymes, le long des routes de pèlerinage à l'époque d'Edo. 


\section{Christophe Marquet}

Christophe Marquet, historien de l'art, est directeur de I'École française d'Extrême-Orient et membre de I'Institut français de recherche sur I'Asie de l'Est, après avoir été professeur des universités à I'INALCO. Ses recherches portent sur I'histoire de l'art et du livre au Japon aux époques moderne et prémoderne. Il a été commissaire de l'exposition Ōtsu-e : peintures populaires du Japon. Des imagiers du XVII siècle à Miró (Paris, Maison de la Culture du Japon, 2019), présentée au Japon sous le titre Peintures d'Ōtsu : regards européens (Ōtsu City Museum of History, 2019).

\section{NOTES}

1. Kusunose Nichinen, "Ōtsu-e no hanashi » [Propos sur les images d'Ōtsu], dans Bi no kuni, vol. 3, n 4, mai 1927, cité dans Christophe Marquet, Ōtsu-e : imagerie populaire du Japon, Arles, Éd. Picquier, 2015, p. 51.

2. Voir Christophe Marquet, "La conception des beauxarts à l'époque de Meiji ", dans Meiji. Splendeurs du Japon impérial, cat. exp. (Paris, musée national des arts asiatiques - Guimet, 2018), Paris, Liénart éditions, 2018, p. 148-155.

3. "Rainen no Kaiga kyōshinkai kisoku no gi ni tsuki... » [À propos du règlement du Salon national de peinture de l'année prochaine...], dans Ōsaka Asahi shinbun, $1^{\text {er }}$ août 1883, p. 2.

4. Philippe Dagen, Primitivismes : une invention moderne, Paris, Gallimard (coll. "Hors série Connaissance »), 2019. On notera que l'auteur considère que les « arts du Japon ne relèvent pas de la question du primitif » (chap. VI, note 67), notamment car ils étaient à l'écart du processus colonial, ce qui est vrai seulement si I'on s'en tient à leur version la plus académique ou à celle qui a été diffusée en Occident par la mode du japonisme au XIX siècle.

5. Voir les principes énoncés dans sa conférence d'avril 1936 au Peer's Club à Tōkyō et publiée dans Sōetsu Yanagi, Folk-Crafts in Japan, Tōkyō, Kokusai bunka shinkōkai, 1936.

6. Muneyoshi Yanagi, «The Peasant Paintings of Ōtsu, Japan ", dans Eastern Art, vol. II, 1930, p. 4-36.

7. Voir Noriko Aso, "Yanagi: Crafting an Alternative Aesthetic Canon ", dans Public Properties. Museums in Imperial Japan, Durham / Londres, Duke University Press, 2014, p. 151-164.

8. C'est la position de l'historien des sciences Yoshida Mitsukuni (1921-1991), qui considère que la pensée de Yanagi est héritière de l'idéologie agrariste défendue par l'État de Meiji. Voir Kumakura Isao, Mingei no hakken [La découverte de l'art populaire], Tōkyō, Kadokawa shoten, 1978, p. 82 et suiv.

9. Noriko Aso fut l'une des premières à chercher les congruences entre les théories qualifiées de «modernistes réactionnaires » de Yanagi et l'esthétique fasciste ("Mediating the Masses: Yanagi Sōtesu and Fascism », dans Alain Tansman [dir.], The Culture of Japanese Fascism, Durham / Londres, Duke University Press, 2009, p. 115-136). Voir aussi l'interprétation politique du Mingei dans Yuko Kikuchi, Japanese Modernisation and Mingei Theory. Cultural Nationalism and Oriental Orientalism, New York, Routledge Curzon, 2004, et Kim Brandt, Kingdom of Beauty: Mingei and the Politics of Folk Art in Imperial Japan, Durham / Londres, Duke University Press, 2007. On notera que ces trois ouvrages éludent la question picturale dans le discours de Yanagi pour se focaliser sur les textes des années de guerre. À l'inverse de ce type de discours déconstructiviste, d'autres auteurs, comme Nakami Mari dans Yanagi Muneyoshi. "Fukugō no bi » no shisō [Yanagi Muneyoshi. Une pensée de la « beauté plurielle »], Tōkyō, Iwanami shoten (coll. « Iwanami shinsho »), 2013, insistent sur le pacifisme et l'humanisme qui caractérisent la pensée de Yanagi et sur sa valorisation des cultures régionales et " périphériques » du Japon (les Aïnous de Hokkaidō, l'archipel des Ryūkyū), et rappellent ses prises de position courageuses dans le contexte colonial, par exemple pour la défense et la mise en valeur des cultures coréenne et des aborigènes de Taiwan.

10. Voir notamment le numéro spécial de la revue Kōgei (nº 108, janvier 1942) consacré à l'art populaire de la région du Tōhoku, avec un texte de Yanagi (" Mingei to Tōhoku ») traitant de ce sujet, issu d'une conférence de 1939.

11. Voir Julien Schuh, "Synthétisme, primitivisme et éloge de la naïveté : le modèle de l'art populaire au XIX siècle ", dans Pascale Alexandre-Bergues (dir.), L'idée de littérature à l'épreuve des arts populaires (1870-1945), actes du colloque (Champs-sur-Marne, Université Paris-Est - Marne-la-Vallée, 2012), Paris, Classiques Garnier, 2015, p. 23-44.

12. Voir Yanagi Sōetsu, «La beauté en quête de critères " (1954), dans Artisan et inconnu. La beauté dans l'esthétique japonaise, Bernard Leach (adaptation), Mathilde Bellaigue (trad. fra.), Paris, L'Asiathèque, 1992, p 17.

13. Voir Kokuritsu minzoku hakubutsukan (dir.), Shibusawa Keizō botsugo gojū-nen. Yaneura-beya no hakubutsukan / Attic Museum, Kyōto, Tankōsha, 2013, et Damien Kunik, "Des objets et des hommes naissance des collections ethnographiques japonaises chez André Leroi-Gourhan et Shibusawa Keizō ", dans Christophe Marquet, Arnaud Nanta, Laurent Nespoulous (dir.), Ebisu, n 52, 2015, p. 199-231.

14. Voir à ce sujet la thèse de doctorat d'Alice Berthon, Le Japon au musée. Le musée national d'ethnologie et le musée national d'histoire et de folklore : histoire comparée et enjeux, Paris, INALCO, 2017, p. 37-40 et p. 170-174.

15. Texte de Yanagi en tête du numéro spécial de la revue Kōgei ( ${ }^{\circ} 17$, mai 1932) consacré aux plaquettes votives de chevaux du XVIII e siècle, conservées à la chapelle dédiée à la déesse Kannon (Okada kannondō) à Hachinohe, au Nord du Japon, avec notamment une contribution de Serizawa Keisuke. Voir aussi sur ce sujet Yanagi Muneyoshi, "Ema », dans Mingei zukan, vol. 2, Tōkyō, Hōbunkan, 1961.

16. Ces deux livres sont Yanagi Muneyoshi, Shoki Ōtsu-e [Les peintures d'Ōtsu de la période primitive], Tōkyō, Kōseikai shuppanbu, 1929, et Yanagi Muneyoshi (dir.), 
Ōtsu-e zuroku [Album de peintures d'Ōtsu], Tōkyō, Sansaisha, 1960.

17. Le premier noyau de cette collection, constitué par Yanagi dans les années 1920, était composé d'une vingtaine de peintures d'Ōtsu et fut exposé pour la première fois en 1927 (galerie Kyūkyodō, Ginza, Tōkyō, environ 10 pièces) et en 1929 (Daimai kaikan, Kyōto, 21 pièces). Cette collection ne cessa par la suite de s'enrichir, au point de représenter désormais un ensemble d'environ 150 pièces, conservées au Japan Folk Crafts Museum de Tōkyō, soit un quart du corpus identifié à ce jour dans les musées japonais. Voir Ogyū Shinzō (dir.), Ōtsu-e. Nihon mingeikan shozō / Ōtsu-e: The Japan Folk Crafts Museum Collection, Ōsaka, Tōhō shuppan, 2005.

18. Voir Christophe Marquet, "Le discours de Yanagi Sōetsu sur la "peinture populaire". Des peintres révolutionnaires à la révolution picturale », dans Cipango, no 16, 2009, p. 43-72.

19. William Morris, "L'art et l'artisanat d'aujourd'hui » (1889), dans L'art et l'artisanat, Thierry Gillyboeuf (trad. fra. et préface), Paris, Payot \& Rivages, 2011, p. 21.

20. Yanagi Muneyoshi, « Ōtsu-e no hanashi » (1929), dans Yanagi Muneyoshi zenshū, vol. 13, Tōkyō, Chikuma shobō, 1982, p. 20.

21. Yanagi Muneyoshi, "Ōtsu-e no hanashi », dans Sansai, nº 63, juin 1954 ; Yanagi, 1982, cité n. 20, p. 256.

22. Pour une présentation générale de ce genre pictural, voir Marquet, 2015, cité n. 1 et Christophe Marquet (dir.), Ōtsu-e : peintures populaires du Japon. Des imagiers du XVII siècle à Miró, cat. exp. (Paris, Maison de la Culture du Japon, 2019), Paris, MCJP / EFEO, 2019.

23. Yanagi, 1982, cité n. 20, p. 22.

24. Voir Christophe Marquet, «Le regard de Nagai Kafū : une relecture des arts d'Edo au début du XX siècle ", dans Cipango, nº 12, 2005, p. 308-329.

25. Christophe Marquet (dir.), Bijutsu fōramu 21, vol. 36, Purimitību kaiga? Kingendai wo ikiru Ōtsu-e, Kyōto, novembre 2017.

26. Kishida Ryūsei, Eiri. Shoki nikuhitsu ukiyo-e [Les peintures ukiyo-e de la période primitive. Ouvrage illustré], Tōkyō, Iwanami shoten, 1926.

27. Yoshikawa Kanpō, Kaiga ni mietaru yōkai [Les créatures surnaturelles dans la peinture], 2 vol., Tōkyō, Bijutsu tosho shuppanbu, 1925-1926. Notons que c'est la même année que le couple de peintres allemands Oscar et Cäcilie Graf publia le premier ouvrage en langue occidentale consacré aux yōkai, dans l'œuvre des grands maîtres de l'ukiyo-e du premier XIXe siècle (Hokusai, Kuniyoshi, Kyōsai, Yoshitoshi, etc.) : Japanisches Gespensterbuch, Stuttgart, Union Deutsche Verlagsgesellschaft, 1925.

28. Hosokibara Seiki, Nihon manga-shi [Histoire du dessin caricatural au Japon], Tōkyō, Yūzankaku, 1924.

29. Hugo Munsterberg, The Folk Arts of Japan, Vermont/ Tōkyō, Charles E. Tuttle Co., 1958, p. 121.

30. Munsterberg, 1958, cité n. 29, p. 19.

31. Voir à ce propos Michael Lucken, "À la poursuite infinie des désirs intérieurs : Yangi Sōetsu avant le Mingei », dans Cipango, no 16, 2009, p. 23-41.
32. Voir notamment l'article de Yanagi, " Kakumei no gaka » [Les peintres révolutionnaires], dans Shirakaba, vol. 3, n 1, janvier 1912, dans lequel il loue de manière inconditionnelle le primitivisme de Gauguin, en citant deux œuvres reproduites en ouverture de la revue, Le cheval blanc (1898, musée d'Orsay), réalisée lors du deuxième séjour à Tahiti, et Aux îles Marquises (vers 1902), ainsi que son célèbre carnet Noa Noa (1901) : "[F]inalement, lassé par la civilisation artificielle de I'Europe, il en vint à aspirer et à rêver de paysages tropicaux primitifs et débordants de couleurs. [...] La touche et la composition [de ses œuvres] sont l'image même de la nature, telle qu'elle se reflète aux yeux d'un nouveau-né. C'est aussi la raison pour laquelle sa peinture est en aplat et primitive. » La revue organisa la même année, en avril 1912, à la bibliothèque municipale de Kyōto, sa cinquième exposition, en présentant plusieurs reproductions photographiques d'œuvres de Gauguin, dont à nouveau Le cheval blanc et Arearea [Joyeusetés] (1892, musée d'Orsay), réalisée lors du premier séjour de l'artiste à Tahiti. Le mois précédent, en avril, la même bibliothèque accueillait I'une des toutes premières expositions de peintures d'Ōtsu, ce qui indique le caractère contemporain de la découverte de ces peintures primitives et des œuvres des post-impressionnistes.

33. Voir Marquet, 2019, cité n. 22, p. 17-18.

34. Voir Christophe Marquet, "Yanagi Sōetsu et l'invention des arts populaires : remise en perspective", dans Cipango, n० 16, 2009, p. 13-22.

35. Kurata Takashi (dir.), " Mingei » no ressun. Tsutanasa no gihō, Tōkyō, Firumuātosha, 2012.

36. Voir en particulier dans le catalogue les essais de Moriya Miho, "Ōtsu-e saikō. Kindai gaka no shiten de miru Ōtsu-e no igi » [Reconsidérer les peintures d'Ōtsu. Le sens des peintures d'Ōtsu du point de vue des artistes modernes] et de Christophe Marquet, « Umi wo koeta Ōtsu-e no miryoku » [Une fascination pour les peintures d'Ōtsu qui s'étendit au-delà des mers].

37. Pour une approche de la nouvelle histoire de l'art proposée par Tsuji Nobuo, on pourra lire, en traduction : Autoportrait de l'art japonais, Strasbourg, Fleur de Parole, 2011 et History of Art in Japan, Tōkyō, University of Tōkyō Press, 2018.

38. Nouvelle édition augmentée : Shinpan. Kisō no keifu, Tōkyō, Shōgakukan, 2019. Traduit sous le titre Lineage of Eccentrics: Matabei to Kuniyoshi, Tōkyō, Kaikai kiki Co., 2012.

39. Pour reprendre le titre de la critique de l'ouvrage ("Seitō-ha bijutsu-shi ni chōsen ») publiée par Takashina Shūji dans I'Asahi shinbun, 21 avril 1970, p. 21.

40. Tsuji Nobuo, «Un porte-drapeau du japonisme en 2018, Itō Jakuchū ", dans Manuela Mosciatello, Aya Ōta (dir.), Jakuchū 1716-1800. Le Royaume coloré des êtres vivants, cat. exp. (Paris, Petit Palais, 2018), Paris, Paris Musées, 2018, p. 48.

41. Tsuji Nobuo et al. (dir.), Nihon bijutsu zenshū, 20 vol., Tōkyō, Shōgakukan, 2013-2016. Un des quatre volumes sur la peinture d'Edo est consacré à Jakuchū, à Ōkyo et aux "peintres excentriques de la capitale » (miyako no kisō). 
42. Tsuji Nobuo, Murakami Takashi, Battle Royale! Japanese Art History, Tōkyō, Kaikai kiki Co., 2017 [éd. orig. : Nettō/Batoru royaru! Nippon bijutsu-shi, Tōkyō, Shinchōsha, 2014].

43. Anne Nishimura Morse (dir.), Takashi Murakami: Lineage of Eccentrics. A collaboration with Nobuo Tsuji and the Museum of Fine Arts, Boston, Boston, MFA Publications, 2018.

44. Kaneko Nobuhisa, Oto Yumiko (dir.), Kawaii Edo kaiga / Cute Edo Paintings, cat. exp. (Tōkyō, Fuchūshi bijutsukan), Tōkyō, Kyūryūdō, 2013.

45. Yajima Arata (dir.), Soboku-bi no keifu. Edo kara Taishō, Shōwa he [Généalogie de la beauté primitive. D’Edo à Taishō et Shōwa], cat. exp. (Tōkyō, Shibuya kuritsu Shōtō bijutsukan), Tōkyō, Shibuya kuritsu Shōtō bijutsukan, 2008.

46. Hokkori bijutsukan [Le musée de l'art apaisant], cat. exp. (Yokosuka Museum of Art, 2015), Shimoda, Yokosuka bijutsukan, 2015.

47. Voir Yajima Arata, Kinsei shūkyō bijutsu no sekai [L'univers de l'art religieux à l'époque prémoderne], Tōkyō, Kokusho kankōkai, 2008.

48. Shirato Shintarō (dir.), Tsukishima, Karukaya. Soboku hyōgen no emaki to setsuwa-ga / Tsukishima Karukaya: The World of Naive Art from Picture Scrolls and Narrative Paintings, cat. exp. (Tōkyō, Nihon Mingeikan), Tōkyō, Nihon Mingeikan, 2013.

49. Yanagi, 1982, cité n. 20, p. 400-422.

50. Jean Cuisenier, L'art populaire en France. Rayonnement, modèles et sources, Paris, Arthaud, 1975, p. 109.

51. Dans une lettre à Langdon Warner, conservateur du Fogg Art Museum, qui I'invita en 1929 à donner un cours d'histoire de l'art du Japon à l'université de Harvard, Yanagi expliqua : "I am not a scholar of the history of art. What I can do, perhaps to a certain extent is to explain the spirit of the East which is manifested in her religion, philosophy and art, so that the interpretation will be more spiritual than historical. » Lettre du 12 avril 1929, dans Yanagi Muneyoshi zenshū, vol. 21, Tōkyō, Chikuma shobō, 1989, p. 600.

52. Heinz-Norbert Jocks, "Expression without Expression. Ten Days Travelling with Sadaharu Horio ", dans Sahaharu Horio, Bruxelles, Vervoordt Foundation / ASA Publishers, 2011, p. 180-181.

53. Life \& Work of Hamada Shōji from the Horio Mikio Collection, Ōsaka, Ōsaka shiritsu tōjiki bijutsukan, 2000.

54. Gravures de Shūji Hisaki. Voir Sadaharu Horio, 2011, cité n. 52, p. 24-27, p. 86-87, p. 98 et 111.

55. Yanagi consacra, dans les années 1950, une série de textes à ces paysans dévots amidistes. Voir Jugaku Bunshō, Yanagi Muneyoshi Myōkōnin-den ronshū, Tōkyō, Iwanami bunko, 1991.

56. Voir "Kōgei ni okeru jiriki-dō to tariki-dō " [La voie de la force individuelle et la voie de la force tierce dans les arts manuels], dans Kōgei, n 12, décembre 1931. Yanagi traduira ces notions par « road of individual development " et "road of humility ", dans son journal de voyage aux États-Unis en 1953, publié dans Yanagi Muneyoshi to Bānādo Rīchi ōfuku shokan.
Nihon mingeikan shiryō-shū, Okamura Mieko, Suzuki Sadahiro (éd.), Tōkyō, Nihon mingeikan, 2014, p. 365. 